\title{
Algoritmos de Conformação de Feixe Baseados na Combinação Convexa de Filtros Adaptativos para Arranjos de Antenas Inteligentes
}

\author{
Wilander Testone Pereira da Silva*, Antônio Henrique dos Santos Ribeiro**.
}

\author{
*Universidade Federal do Maranhão, São Luís, MA, Brasil, Departamento de Engenharia de Eletricidade, Laboratório de \\ Sistemas Adaptativos e Processamento de Sinais (e-mails: wi lander028 ( hotmai I.com). \\ **Universidade Federal do Maranhão, São Luís, MA, Brasil, Departamento de Engenharia de Eletricidade, Laboratório de \\ Controle Inteligente e Sistemas Embarcados (e-mail: scfcahenriquer@gmai I . com).
}

\begin{abstract}
This paper proposed two novel beamforming algorithms using convex combination of adaptive filters for smart antenna array. The proposed method consists of applying two individual filters convexly combined through a mixture parameter whose update is based on a normalized adaptation scheme associated to a sigmoidal function. The convergence of the CNLMS-CLMS and CNLMS-IPNLMS algorithms are analyzed in terms of mean-square error (MSE). Numerical simulations results show that the performance in terms of convergence speed of the CNLMS-CLMS and CNLMS-IPNLMS convex combinations is superior to the performance of IPNLMS, CNLMS and CLMS algorithms. We show that the convergence of proposed algorithms presents low sensitivity to variations in both the input signal-to-interference-plus-noise ratio, number of antenna elements as well as step size.
\end{abstract}

Resumo: Este artigo propõe dois novos algoritmos de conformação de feixe usando combinação convexa de filtros adaptativos para arranjos de antenas inteligentes. 0 método proposto, consiste em utilizar dois filtros individuais combinados convexamente através de um parâmetro de mistura, cuja atualização é baseada em um esquema de adaptação normalizada, associada a uma função sigmoide. A convergência dos algoritmos CNLMS-CLMS e CNLMS-IPNLMS é analisada em termos do erro quadrático médio (MSE). Resultados de simulações numéricas mostram que o desempenho em termos de velocidade de convergência das combinações convexas CNLMS-CLMS e CNLMS-IPNLMS é superior ao desempenho dos algoritmos IPNLMS, CNLMS e CLMS. Mostra-se que a convergência dos algoritmos propostos apresenta baixa sensibilidade às variações na relação sinal-interferência mais ruído, no número de elementos das antenas bem como no tamanho do passo.

Keywords: Beamforming algorithms, convex combination, adaptive filters, MSE.

Palavras-chaves: Algoritmos de conformação de feixe, combinação convexa, filtros adaptativos, MSE.

\section{INTRODUÇÃO}

Os sistemas de comunicação sem fio atualmente estão à procura de novas tecnologias a serem implementadas em seu ambiente, portanto, buscam melhoria e aumento da qualidade do sinal. Para esta proposta, o uso de antenas inteligentes torna-se predominante para o sistema. Neste ponto, associa-se a importância dos filtros adaptativos com os esquemas de combinação convexa (OrozcoTupacyupanqui et al., 2015) e (Orozco-Tupacyupanqui et al., 2016) utilizando os algoritmos CLMS (Andrade et al., 2012) CNLMS (Andrade et al., 2013) e IPNLMS (Benesty and Gay, 2002) para a conformação de feixe em um arranjo adaptativo (Srar et al., 2010). Os resultados apresentados

\footnotetext{
O presente trabalho foi realizado com apoio da Fundação de Amparo à Pesquisa e Desenvolvimento Científico do Maranhão (FAPEMA), da Coordenação de Aperfeiçoamento de Pessoal de Nível Superior - Brasil (CAPES) - Código de Financiamento 001 e do Conselho Nacional de Desenvolvimento Científico e Tecnológico (CNPq).
}

da investigação de convergência dos algoritmos clássicos e propostos avaliam o comportamento do filtro adaptativo pelas variações de parâmetros na etapa de atualização, número de elementos das antenas e relação sinal ruído mais interferência para os referidos filtros.

Em (Andrade et al., 2015) e (Korayem and Bendoukha, 2018), são apresentados um levantamento e uma análise comparativa do desempenho dos algoritmos LMS restritos e suas variantes para resolver problemas relacionados com a conformação de feixe em arranjo adaptativo. Esperase que os arranjos de antenas inteligentes proporcionem um desempenho melhor do que as antenas habituais existentes em termos de consumo de energia, capacidade do usuário e supressão de ruído (Alexiou and Haardt, 2004), (Boukalov and Haggman, 2000). Essas vantagens devem-se aos arranjos de antenas e a técnicas de desempenho no processamento de sinais digitais (DSP) usados no sistema. As antenas inteligentes suportadas pela capacidade de processamento de sinal usam um feixe estreito no ponto de direção para os usuários desejados, 
mas ao mesmo tempo introduz nulo na direção da interferência, otimizando assim a qualidade do serviço e capacidade de transmissão (Moghaddam et al., 2010).

A capacidade dessas antenas de rastrear seus sinais alvo com rapidez e precisão depende muito do desempenho do algoritmo de conformação de feixe empregado. Entre muitos algoritmos adaptativos estudados, o algoritmo CLMS (constrained LMS) (Frost, 1972) oferece implementação mais simples e boa capacidade de rastreamento, enquanto os algoritmos CNLMS (constrained NLMS) (Apolinário et al., 1998) e IPNLMS (improved proportionate normalized LMS) (Liu et al., 2008) fornecem uma convergência relativamente rápida. Motivado pelo esquema de adaptação normalizada para combinação convexa de dois filtros adaptativos (Azpicueta-Ruiz et al., 2008) e pelo bom resultado apresentado em (Srar et al., 2010), o trabalho aqui apresentado concentrou-se em algoritmos de adaptação linearmente restritos (CLMS/CNLMS) e no algoritmo IPNLMS. As principais características que distinguem os algoritmos propostos dos algoritmos CLMS, CNLMS e IPNLMS são: taxa de convergência para o erro quadrático médio (MSE) quando aplicado a conformação de feixe adaptativo.

\section{ARRANJO DE ANTENAS}

Seja um arranjo linear uniforme (ULA) composto por $N$ antenas e $i-1$ recebendo sinais de banda estreita vindos de diferentes direções $x_{0} \ldots x_{i-1}$. 0 vetor do sinal de entrada é descrito como

$$
\mathbf{x}(n)=\sum_{m=0}^{i-1} \mathbf{x}_{m}(n) \mathbf{S}(\theta)+\boldsymbol{\varphi}(n)
$$

onde $\mathbf{S}(\theta)=\left[1, e^{j i 2 \pi d \cos \theta}, \ldots, e^{j(i-1) 2 \pi d \cos \theta}\right]^{T}$ é a matriz de direcionamento, com $d$ sendo a distância entre os elementos da antena. Por (1), o vetor do sinal de interesse é $\mathbf{x}_{S}(n)=\mathbf{x}_{0}(n) \mathbf{S}(\theta)$ e $\mathbf{x}_{I}(n)=\sum_{m=1}^{i-1} \mathbf{x}_{1}(n) \mathbf{S}(\theta)$ é o vetor do sinal de interferência, $\operatorname{com} \varphi(n)$ sendo vetor do sinal de ruído.

A partir da formulação acima e impondo restrições lineares, é possível obter uma expressão de forma fechada para o ótimo $\mathbf{w}^{*}$ (Andrade et al., 2012), definido por

$$
\mathbf{w}^{*}=\left(\mathbf{R}_{x x}\right)^{-1} \mathbf{C}\left(\mathbf{C}^{H}\left(\mathbf{R}_{x x}\right)^{-1} \mathbf{C}\right)^{-1} \mathbf{z},
$$

onde $\mathbf{R}_{x x}$ é a matriz de autocorrelação e $\mathbf{C}^{H} \mathbf{w}=\mathbf{z}$, com $\mathbf{C}$ dado por

$$
\left[1, e^{j(i-1) 2 \pi d \operatorname{sen}\left(\theta_{i}\right)}, \cdots, e^{j(i-1) 2 \pi d(M-1) \operatorname{sen}\left(\theta_{i}\right)}\right]^{T} .
$$

O ângulo $\theta_{i}$ representa as direções dos sinais de interesse e interferência. Aqui, consideramos uma onda plana com espaçamento $d$ incidindo de $\theta$, o padrão de feixe de uma antena isotrópica é dado por

$$
A F(\theta)=\sum_{i=0}^{M-1} w_{i} e^{j(i-1) 2 \pi d \operatorname{sen}(\theta)}
$$

\section{ALGORITMOS DE CONFORMAÇÃO DE FEIXE}

Nesta Seção, são apresentados os principais aspectos da implementação dos algoritmos CLMS, CNLMS e IPNLMS, com foco nos aspectos de convergência e formulação, bem como, a aproximação do sinal desejado e sua velocidade, tendo em conta as restrições no tempo das aplicações para atender a demanda no ambiente online.

\subsection{Algoritmo LMS Restrito}

O algoritmo CLMS foi proposto por (Frost, 1972) para resolver problemas relacionados a arranjo de antenas, cujas soluções poderiam comtemplar a direção do sinal de interesse e ao mesmo tempo atenuar sinais interferentes de outras direções. O CLMS pode ser formulado como

$$
\min _{\mathbf{w}} E\left[|e(n)|^{2}\right] \quad \text { sujeito } a \quad \mathbf{C}^{H} \mathbf{w}=\mathbf{z},
$$

com $\mathbf{C}$ sendo a matriz restrita e $\mathbf{z}$ o vetor de restrição. Usando as técnicas dos multiplicadores de lagrange, temos

$$
\varepsilon(\mathbf{w})=E\left[|e(n)|^{2}\right]+\mathbf{\kappa}_{1}^{\mathrm{H}}\left(\mathbf{C}^{\mathrm{H}} \mathbf{w}-\mathbf{z}\right) .
$$

A função custo $\varepsilon(\mathbf{w})$ pode ser minimizada aplicando o método de descida mais íngreme, tal qual o vetor peso é atualizado a cada iteração

$$
\mathbf{w}(n+1)=\mathbf{w}(n)-\frac{\mu}{2} \nabla_{\mathbf{w}} \varepsilon(\mathbf{w}),
$$

empregando as manipulações algébricas e encontrando o valor de $\boldsymbol{\kappa}_{1}$ para $\widehat{\nabla}_{\mathbf{w}} \varepsilon(\mathbf{w})=-2 e^{*}(n) \mathbf{x}(n)+\mathbf{C} \boldsymbol{\kappa}_{1}=0$, temse

$$
\mathbf{w}(n+1)=\mathbf{P}_{\mathrm{a}}\left[\mathbf{w}(n)+\mu e^{*}(n) \mathbf{x}(n)\right]+\mathbf{w}_{\mathrm{a}},
$$

onde $\mathbf{P}_{\mathrm{a}}=\mathbf{I}-\mathbf{C C}^{H} \mathbf{C C}^{H^{-1}}$ é uma matriz de projeção, $\mu$ é o parâmetro do passo e $\mathbf{w}_{\mathrm{a}}=\mathbf{C}\left(\mathbf{C C}^{H}\right)^{-1} \mathbf{z}$ é um vetor $N \mathrm{x} 1$.

\subsection{Algoritmo NLMS Restrito}

0 algoritmo NLMS restrito ou (CNLMS) é a versão normalizada do CLMS. Pode ser derivado se minimizarmos o erro instantâneo a posteriori no instante $n$ (Apolinário et al., 1998) e (Diniz, 2010)

$$
\frac{\partial e_{\mathrm{ap}}(n) e_{\mathrm{ap}}^{*}(n)}{\partial \mu^{*}}=0,
$$

com $e_{\text {ap }}(n)=d_{n}-\mathbf{w}_{n+1}^{H} \mathbf{x}_{n}=e_{n}\left(1-\mu \mathbf{x}_{n}^{H} \mathbf{P}_{\mathrm{a}} \mathbf{x}_{n}\right)$. Por (Andrade et al., 2015), tem-se:

$$
\frac{\partial e_{\mathrm{ap}}(n) e_{\mathrm{ap}}^{*}(n)}{\partial \mu^{*}}=\frac{e_{\mathrm{ap}}^{*}(n)}{2}\left\{\frac{\partial e_{\mathrm{ap}}(n)}{\partial\left(R \mu^{*}\right)}+j \frac{\partial e_{\mathrm{ap}}(n)}{\partial\left(I \mu^{*}\right)}\right\}
$$

usando Cauchy-Riemann (Brandwood, 1983), obtém-se:

$$
\begin{aligned}
\frac{\partial e_{\mathrm{ap}}(n)}{\partial\left(R \mu^{*}\right)} & =\frac{1}{2}\left[e_{n} \frac{\partial\left(1-\mu \mathbf{x}_{n}^{H} \mathbf{P}_{\mathrm{a}} \mathbf{x}_{n}\right)^{*}}{\partial\left(R \mu^{*}\right)}+e_{n}^{*} \frac{\partial\left(1-\mu \mathbf{x}_{n}^{H} \mathbf{P}_{\mathrm{a}} \mathbf{x}_{n}\right)}{\partial\left(R \mu^{*}\right)}\right] \\
& =-\frac{1}{2}\left[e_{n}\left(-\mathbf{x}_{n}^{H} \mathbf{P}_{\mathrm{a}} \mathbf{x}_{n}\right)^{*}+e_{n}^{*}\left(-\mathbf{x}_{n}^{H} \mathbf{P}_{\mathrm{a}} \mathbf{x}_{n}\right)\right]
\end{aligned}
$$

onde $w_{i}$ é o i-ésimo componente do vetor de peso $\mathbf{w}$. 
$\frac{\partial e_{\mathrm{ap}}(n)}{\partial\left(R \mu^{*}\right)}=-\frac{1}{2}\left[-e_{n}\left(\mathbf{x}_{n}^{H} \mathbf{P}_{\mathrm{a}} \mathbf{x}_{n}\right)^{*}-e_{n}^{*}\left(\mathbf{x}_{n}^{H} \mathbf{P}_{\mathrm{a}} \mathbf{x}_{n}\right)\right]$

e

$$
\begin{aligned}
\frac{\partial e_{\mathrm{ap}}(n)}{\partial\left(I \mu^{*}\right)} & =\frac{1}{2}\left[e_{n} \frac{\partial\left(1-\mu \mathbf{x}_{n}^{H} \mathbf{P}_{\mathrm{a}} \mathbf{x}_{n}\right)^{*}}{\partial\left(I \mu^{*}\right)}+e_{n}^{*} \frac{\partial\left(1-\mu \mathbf{x}_{n}^{H} \mathbf{P}_{\mathrm{a}} \mathbf{x}_{n}\right)(n)}{\partial\left(I \mu^{*}\right)}\right] \\
& =\frac{j}{2}\left[e_{n}\left(-\mathbf{x}_{n}^{H} \mathbf{P}_{\mathrm{a}} \mathbf{x}_{n}\right)^{*}-e_{n}^{*}\left(-\mathbf{x}_{n}^{H} \mathbf{P}_{\mathrm{a}} \mathbf{x}_{n}\right)\right] \\
\frac{\partial e_{\mathrm{ap}}(n)}{\partial\left(I \mu^{*}\right)} & =j * \frac{1}{2}\left[-e_{n}\left(\mathbf{x}_{n}^{H} \mathbf{P}_{\mathrm{a}} \mathbf{x}_{n}\right)^{*}+e_{n}^{*}\left(\mathbf{x}_{n}^{H} \mathbf{P}_{\mathrm{a}} \mathbf{x}_{n}\right)\right] .
\end{aligned}
$$

A derivada da função custo $e_{\mathrm{ap}}(n)$ com relação a $\mu^{*}$, é definida como:

$$
\begin{aligned}
\frac{\partial\left(e_{\mathrm{ap}}(n)\right)}{\partial \mu^{*}} & =\frac{1}{2}\left[\frac{1}{2} e_{n}\left(\mathbf{x}^{H} / \mathbf{P}_{\mathrm{a}} \mathbf{x}_{n}\right)^{*}+\frac{1}{2} e_{n}^{*}\left(\mathbf{x}_{n}^{H} \mathbf{P}_{\mathrm{a}} \mathbf{x}_{n}\right)\right. \\
& \left.-\frac{1}{2} e_{n}\left(\mathbf{x}_{n}^{H} \mathbf{a}_{\mathrm{a}}^{*} \mathbf{x}_{n}\right)^{*} j+\frac{1}{2} e_{n}^{*}\left(\mathbf{x}_{n}^{H} \mathbf{P}_{\mathrm{a}} \mathbf{x}_{n}\right) j\right] \\
& =\frac{1}{2}\left[\frac{1}{2} e_{n}^{*}\left(\mathbf{x}_{n}^{H} \mathbf{P}_{\mathrm{a}} \mathbf{x}_{n}\right)+\frac{1}{2} e_{n}^{*}\left(\mathbf{x}_{n}^{H} \mathbf{P}_{\mathrm{a}} \mathbf{x}_{n}\right) j\right] \\
\frac{\partial\left(e_{\mathrm{ap}}(n)\right)}{\partial \mu^{*}} & =\frac{1}{2}\left[\left(e_{n}^{*} \mathbf{x}_{n}^{H} \mathbf{P}_{\mathrm{a}} \mathbf{x}_{n}\right)\right] .
\end{aligned}
$$

Tomando (10) e resolvendo (9), para $\mu$, tem-se:

$$
\begin{gathered}
\frac{\left[e_{n}\left(1-\mu \mathbf{x}_{n}^{H} \mathbf{P}_{\mathrm{a}} \mathbf{x}_{n}\right)\right]^{*}}{2} * \frac{e_{n}^{*} \mathbf{x}_{n}^{H} \mathbf{P}_{\mathrm{a}} \mathbf{x}_{n}}{2}=0 \\
=\left(e_{n}^{2}\right)^{*} \mathbf{x}_{n}^{H} \mathbf{P}_{\mathrm{a}} \mathbf{x}_{n}-\mu e_{n}^{*} \mathbf{x}_{n}^{*} \mathbf{P}_{\mathrm{a}} \mathbf{x}_{n} *\left(e_{n}^{*} \mathbf{x}_{n}^{H} \mathbf{P}_{\mathrm{a}} \mathbf{x}_{n}\right)=0 \\
\left(e_{n}^{2}\right)^{*} \mathbf{x}_{n}^{H} \mathbf{P}_{\mathrm{a}} \mathbf{x}_{n}=\mu e_{n}^{*} \mathbf{x}_{n}^{*} \mathbf{P}_{\mathrm{a}} \mathbf{x}_{n} *\left(e_{n}^{*} \mathbf{x}_{n}^{H} \mathbf{P}_{\mathrm{a}} \mathbf{x}_{n}\right) \\
(e /)^{*} \mathbf{x}_{n}^{H} \mathbf{P}_{\mathrm{a}} \mathbf{x}_{n}=\mu\left(e_{n}\right)^{*}\left(\mathbf{x}_{n}^{H}\right)^{2} \mathbf{P}_{\mathrm{a}}^{2} \mathbf{x}_{n}^{2} \\
\mu=\frac{1}{\mathbf{x}_{n}^{H} \mathbf{P}_{\mathrm{a}} \mathbf{x}_{n}} .
\end{gathered}
$$

A fórmula da forma fechada em (Apolinário etc. al., 1998) do algoritmo CNLMS, é dada a partir da Equação (8), portanto

$$
\mathbf{w}(n+1)=\mathbf{P}_{\mathrm{a}}\left[\mathbf{w}(n)+\frac{e^{*}(n) \mathbf{x}(n)}{\mathbf{x}_{n}^{H} \mathbf{P}_{\mathrm{a}} \mathbf{x}_{n}}\right]+\mathbf{w}_{\mathrm{a}} .
$$

Aqui, obtem-se um $\mu_{0}$ para controlar as curvas de aprendizagem no numerador e a soma de um $\varepsilon$ no denominador de (11), sendo um parâmetro constante muito pequeno incluído para evitar o excesso quando $\mathbf{x}_{n}^{H} \mathbf{P}_{\mathrm{a}} \mathbf{x}_{n}$ torna-se também pequeno, assim:

$$
\mu=\frac{1}{\mathbf{x}_{n}^{H} \mathbf{P}_{\mathrm{a}} \mathbf{x}_{n}} \Rightarrow \mu=\frac{\mu_{0}}{\mathbf{x}_{n}^{H} \mathbf{P}_{\mathrm{a}} \mathbf{x}_{n}+\varepsilon}
$$

A nova equação do CNLMS será (Andrade et al., 2015):

$$
\mathbf{w}(n+1)=\mathbf{P}_{\mathrm{a}}\left[\mathbf{w}(n)+\mu_{0} \frac{e^{*}(n) \mathbf{x}(n)}{\mathbf{x}_{n}^{H} \mathbf{P}_{\mathrm{a}} \mathbf{x}_{n}+\varepsilon}\right]+\mathbf{w}_{\mathrm{a}} .
$$

A Equação (14) é chamada de algoritmo NLMS restrito.

\subsection{Algoritmo IPNLMS}

Nesta Seção, o algoritmo IPNLMS é utilizado para a aplicação em conformação de feixe, devido a rápida velocidade de convergência para arranjos esparsos. Ele foi proposto e implementado por (Benesty and Gay, 2002). A sua equação de atualização é dada por

$$
\mathbf{w}(n+1)=\mathbf{w}(n)+\frac{\mu \mathbf{G}(n) \mathbf{x}_{n} e_{n}^{*}}{\mathbf{x}_{n}^{T} \mathbf{G}(n) \mathbf{x}_{n}+\varepsilon}
$$

onde $\mathbf{G}(n)$ é a matriz de ganhos dada por

$$
\mathbf{G}(n)=\operatorname{diag}\left[g_{1}(n) g_{2}(n) \ldots g_{n}(n)\right],
$$

e os ganhos individuais dado por

$$
g_{n}(n)=\frac{1-\alpha}{2}+\frac{N(1+\alpha)\left|\mathbf{w}_{n}(n)\right|}{\left\|\mathbf{w}_{n}\right\|_{1}+\varsigma}
$$

onde $\alpha$ é o parâmetro de proporcionalidade do IPNLMS e $\varsigma$ é uma parâmetro de regularização usado para evitar divisão por zero. A fórmula da forma fechada do algoritmo IPNLMS é dada por (15).

\section{COMBINAÇÃO CONVEXA DE DOIS FILTROS ADAPTATIVOS}

Nesta Seção, a combinação convexa de dois filtros adaptativos é aplicada a conformação de feixe em arranjo de antenas. A estrutura do arranjo inteligente para a combinação convexa de dois filtros é mostrada na Figura 1 e seu algoritmo iterativo modificado segue as subseções abaixo. 0 vetor de entrada do arranjo, $\mathbf{x}(n)$, é a soma ponderada dos sinais recebidos com um ruído aditivo, sendo dado por (1). Neste sistema, os pesos são calculados com base na saída do filtro combinada conectados ao parâmetro de mistura $\lambda(n)$ e ao sinal de referência $d(n)$. Os algoritmos tendem a atenuar os sinais interferentes e obter melhor sinal desejado no sistema.

\subsection{Adaptação Normalizada usando pn-LMS}

O esquema de adaptação normalizada foi introduzido em (Azpicueta-Ruiz et al., 2008) a fim de ser mais robusta às mudanças no cenário de filtragem. Considerando a equação:

$$
e_{i}(n)=d(n)-y_{i}(n),
$$

com $i=1,2$ é possível reescrever

como

$$
y(n)=\lambda(n) y_{1}(n)+[1-\lambda(n)] y_{2}(n)
$$




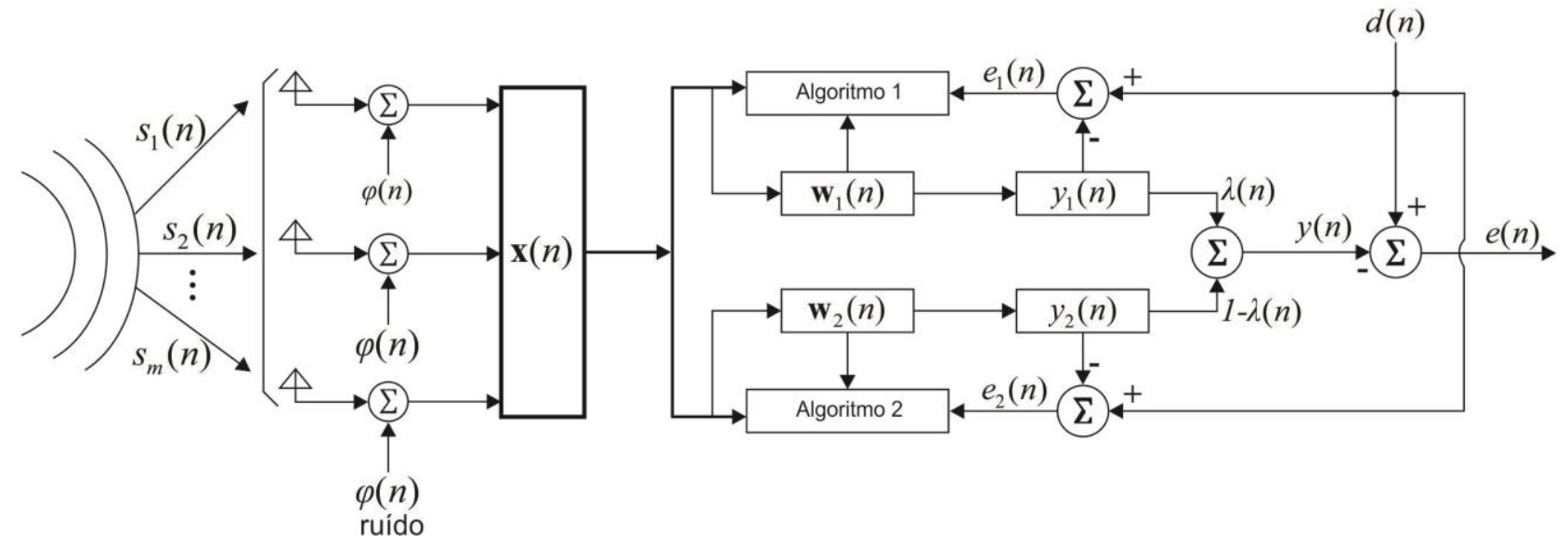

Fig. 1 Arranjo inteligente com estrutura da combinação convexa de dois filtros adaptativos.

$$
\begin{aligned}
& y(n)=\lambda(n) y_{1}(n)+y_{2}(n)-\lambda(n) y_{2}(n) \\
& y(n)=y_{2}(n)+\lambda(n)\left[y_{1}(n)-y_{2}(n)\right] .
\end{aligned}
$$

A equação a seguir:

$$
\begin{aligned}
a(n+1)= & a(n)+\nabla a(n) \\
a(n+1)= & a(n)+\mu_{a} e_{2}\left[y_{1}(n)-y_{2}(n)\right] \\
& \lambda(n)[1-\lambda(n)],
\end{aligned}
$$

mostra que a regra de adaptação existente para o parâmetro de mistura é equivalente ao filtro LMS com tamanho do passo variante $\mu_{a} \lambda(n)[1-\lambda(n)]$ e a saída do filtro $y_{1}(n)-y_{2}(n)$. De fato, esta interpretação é reforçada pelo fato de que a saída do filtro em (20) pode ser reescrita como

$$
y(n)=\lambda(n) y_{1}(n)+[1-\lambda(n)] y_{2}(n),
$$

que podemos pensar no esquema de combinação geral como um filtro adaptativo de duas camadas. Na primeira camada, os dois filtros componentes operam independentemente um dos outros e de acordo com suas próprias regras, enquanto a segunda camada consiste de um filtro com sinal de entrada $e_{2}(n)-e_{1}(n)$ que minimiza o erro geral. Esta interpretação do esquema de combinação sugere que outras vantagens poderiam ser obtidas se usássemos uma regra LMS normalizada para adaptar o parâmetro de mistura do que LMS padrão. Como $e_{2}(n)-$ $e_{1}(n)$ desempenha o papel do sinal de entrada neste nível, faz sentido usar o seguinte esquema de adaptação:

$$
\begin{aligned}
a(n+1)= & a(n)+\frac{\mu_{a} \lambda(n)[1-\lambda(n)]}{\left|e_{2}(n)-e_{1}(n)\right|^{2}} e(n) \\
& {\left[e_{2}(n)-e_{1}(n)\right] . }
\end{aligned}
$$

Na prática, contudo, o desempenho deste esquema é bastante insatisfatório dado que o valor instantâneo $\left[e_{2}(n)-e_{1}(n)^{2}\right]$ é uma estimativa muito pobre da potência do sinal de entrada da "segunda camada". Similarmente, ao algoritmo LMS normalizado com normalização de potência (Sayed, 2008), o melhor comportamento é obtido por

$$
\begin{aligned}
a(n+1)= & a(n)+\frac{\mu_{a}}{r(n+1)} \lambda(n)[1-\lambda(n)] e(n) \\
& {\left[e_{2}(n)-e_{1}(n)\right], }
\end{aligned}
$$

onde

$$
r(n+1)=\beta r(n)+(1-\beta)\left[e_{2}(n)-e_{1}(n)\right]^{2},
$$

é uma estimativa aproximada da potência do sinal de interesse. Aqui, a seleção do fator de esquecimento $\beta$ é bastante sensível na aplicação em conformação de feixe adaptativo.

\subsection{Combinação Proposta para Beamformer usando CNLMS- CLMS e CNLMS-IPNLMS}

Nesta Seção, é considerada uma combinação convexa de filtros adaptativos aplicada a este beamformer, onde a saída no tempo $n$, dada por $y(n)$ é alcançada por uma combinação linear, onde a saída geral do filtro é dada por (Arenas-Garcia et al., 2016)

$$
y(n)=\lambda(n) y_{1}(n)+[1-\lambda(n)] y_{2}(n),
$$

com $y_{i}(n)=\mathbf{x}^{T}(n) \mathbf{w}_{i}(n), i=1,2$, sendo as saídas dos dois filtros adaptativos caracterizados pelos pesos $w_{i}(n)$ e pelo parâmetro de mistura $\lambda(n)$. Similarmente, o vetor de peso do esquema da combinação é dado por

$$
\mathbf{w}(n)=\lambda(n) \mathbf{w}_{1}(n)+[1-\lambda(n)] \mathbf{w}_{2}(n),
$$

onde

$$
e(n)=d(n)-\mathbf{w}^{T}(n) \mathbf{x}(n),
$$

é o erro da componente do filtro adaptativo. 0 parâmetro da combinação $\lambda(n)$ é restrito ao intervalo $[0,1]$ para combinações convexas. 0 parâmetro de mistura, $\lambda(n)$, é dado por

$$
\lambda(n)=\frac{1}{1+e^{-a(n)}},
$$

isto é, $\lambda(n)$ deriva da adaptação de um parâmetro auxiliar, $a(n)$, que é atualizado por meio de (24).

\section{SIMULAÇÕES}

Para avaliar o desempenho dos algoritmos propostos e convencionais são feitas simulações de Monte Carlo (média de 100 realizações) aplicadas a um arranjo de antenas linear uniformemente espaçado com 3 e 32 elementos e $d=0.5 \tau$. Todos os elementos são uniformemente simulados com valores de amplitude fixos. 
Na Tabela 1, são listados os parâmetros dos algoritmos CLMS, CNLMS, IPNLMS, CNLMS-CLMS e CNLMS-IPNLMS, usados para estimar a função valor a cada instante.

Tabela 1. Parâmetros dos algoritmos propostos e convencionais.

\begin{tabular}{|l|c|c|c|c|c|c|c|}
\cline { 2 - 9 } \multicolumn{1}{c|}{} & \multicolumn{7}{c|}{ Configuração dos parâmetros - 3 } \\
\hline Algoritmos & $\rho$ & $\alpha$ & $\delta$ & $\mu$ & $\beta$ & $\lambda$ & $a$ \\
\hline CLMS & - & - & - & 0,08 & - & - & - \\
\hline CNLMS & - & - & - & 0,1 & - & - & - \\
\hline IPNLMS & 1,0 & 0,5 & 0,01 & 0,4 & - & - & - \\
\hline CNLMS-CLMS & - & - & - & $10^{-3}$ & $10^{-4}$ & 0,341 & $-4,0$ \\
\hline CNLMS-IPNLMS & 1,0 & 0,5 & 0,01 & $10^{-3}$ & 0,005 & 0,341 & $-1,5$ \\
\hline & \multicolumn{7}{|c|}{ Configuração dos parâmetros - } \\
\hline Algoritmos & $\rho$ & $\alpha$ & $\delta$ & $\mu$ & $\beta$ & $\lambda$ & $a$ \\
\hline CLMS & - & - & - & 0,01 & & - & - \\
\hline CNLMS & - & - & - & 0,01 & & - & - \\
\hline IPNLMS & 1,0 & 0,5 & 0,01 & 0,35 & & - & - \\
\hline CNLMS-CLMS & - & - & - & $10^{-3}$ & 0,005 & 0,3 & $-0,5$ \\
\hline CNLMS-IPNLMS & 1,0 & 0,5 & 0,01 & $10^{-3}$ & 0,005 & 0,3 & $-1,25$ \\
\hline
\end{tabular}

Na Fig. 2, apresentamos dois cenários para comparação de desempenho do padrão de feixe do arranjo uniformemente espaçado obtido usando os algoritmos CNLMS-IPNLMS, CNLMS-CLMS, IPNLMS, CNLMS, CLMS e LCMV. O cenário I representa o padrão de radiação quando a direção desejada atinge $10^{\circ}$ e dois sinais interferentes são assumidos a ser assumidos a $-40^{\circ}$ e $45^{\circ}$. O cenário II mostra a direção desejada chegando a $0^{\circ}$ e dois sinais interferentes a $25^{\circ}$ e $60^{\circ}$. A relação sinal ruído mais interferência (SINR) é assumida a ser $20 \mathrm{~dB}$ em ambos os

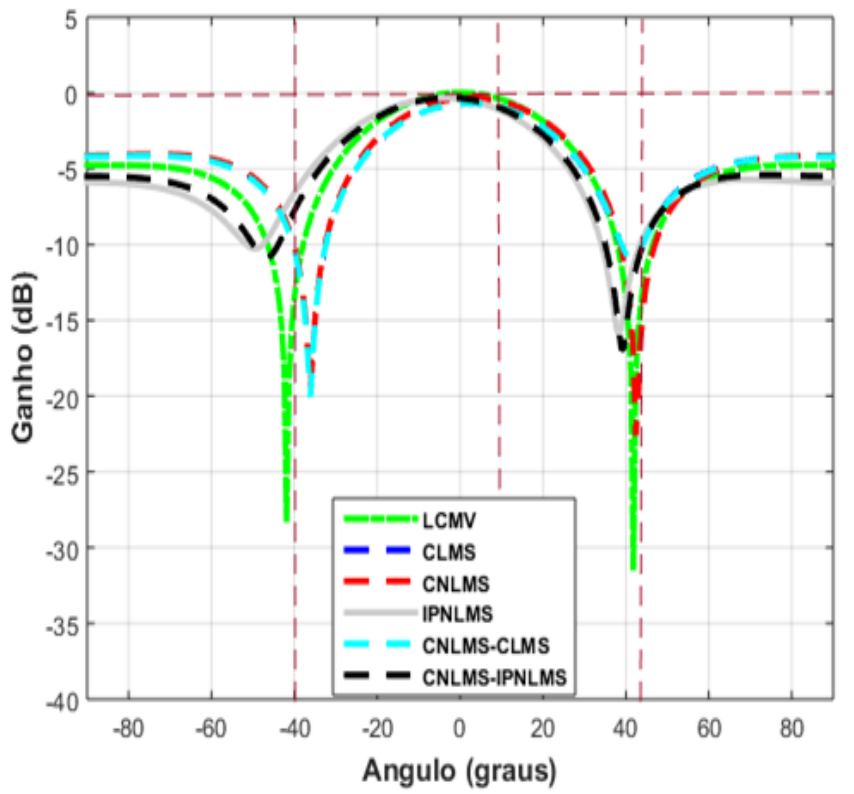

casos. Os arranjos rastreiam o sinal desejado com sucesso e colocam nulos levemente deslocados nas direções dos interferentes. 0 padrão de radiação do arranjo linear usando ambos algoritmos é gerado de acordo com (4). Para ter em conta as restrições lineares usados pelos algoritmos (CNLMS-IPNLMS, CNLMS-CLMS, CNLMS, CLMS e LCMV), eles correspondem a uma direção de interesse e dois sinais interferentes, a matriz $\mathbf{C}$ têm 3 colunas e o vetor $\mathbf{z}$ tem 3 linhas, $\mathbf{z}=\left[\begin{array}{lll}1 & 0 & 0\end{array}\right]^{T}$.

Fig. 3 e 4, descrevem o comportamento dos pesos $\Delta \mathrm{W}_{1-3}$ para 15 iterações dos arranjos de ambos os cenários descritos anteriormente. A análise de desempenho é apresentada pelo comportamento do parâmetro de estimação no vetor peso $\Delta \mathrm{W}$ no ambiente de aprendizagem. Percebe-se das Figuras 3 e 4 uma boa precisão dos modelos propostos e convencionais para descrever o comportamento médio dos coeficientes na adaptação dos algoritmos. Aqui, simulamos a parte real e imaginária dos 3 elementos do arranjo de 3 e 32 antenas.

Fig. 5, simulamos o erro quadrático médio (MSE) em cada iteração. Observa-se que as abordagens propostas apresentam maior velocidade de convergência e menor erro em estado estacionário em relação à variação em SINR (10 dB e $20 \mathrm{~dB})$, tamanho do passo e número de elementos na antena. Neste artigo, usando a combinação convexa de dois filtros adaptativos aplicado a conformação de feixe em arranjo de antenas inteligentes, o ajustamento correto (da SINR, do tamanho do passo, do fator de esquecimento, do parâmetro de mistura e do número de elementos na antena) definem as características do cenário de filtragem. Portanto, o comportamento universal desejado da combinação convexa nem sempre pode ser alcançado, como visto na Fig. 5.

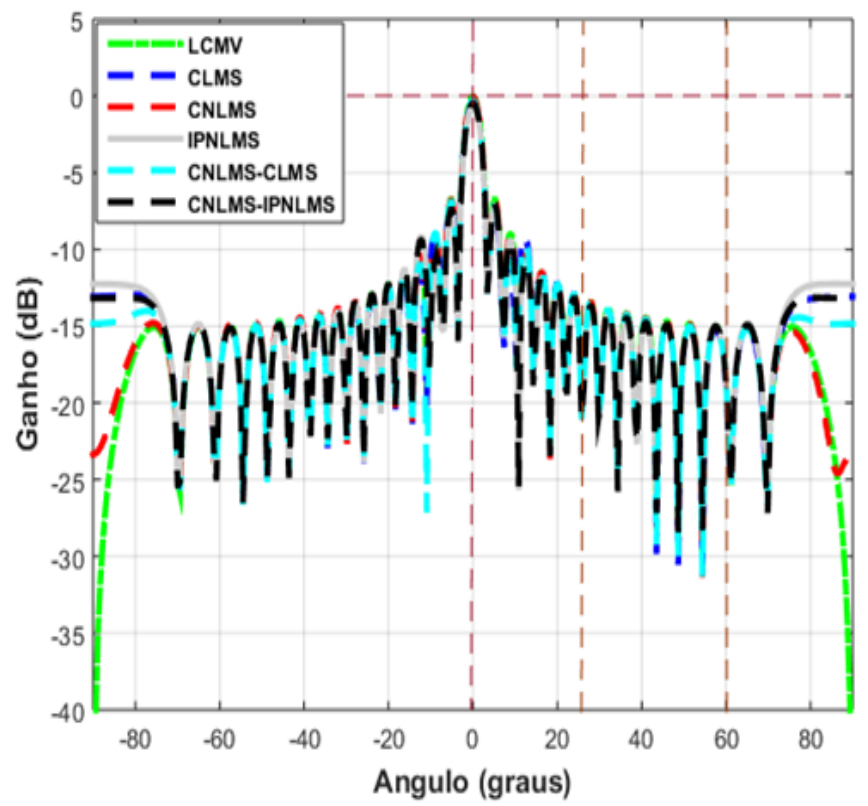

Fig. 2: Padrão de feixe de radiação dos algoritmos LCMV, CLMS, CNLMS, IPNLMS, CNLMS-CLMS e CNLMS-IPNLMS para os casos 3 e 32 elementos na antena com SINR $=20 \mathrm{~dB}$ e $n=100$ iterações. 

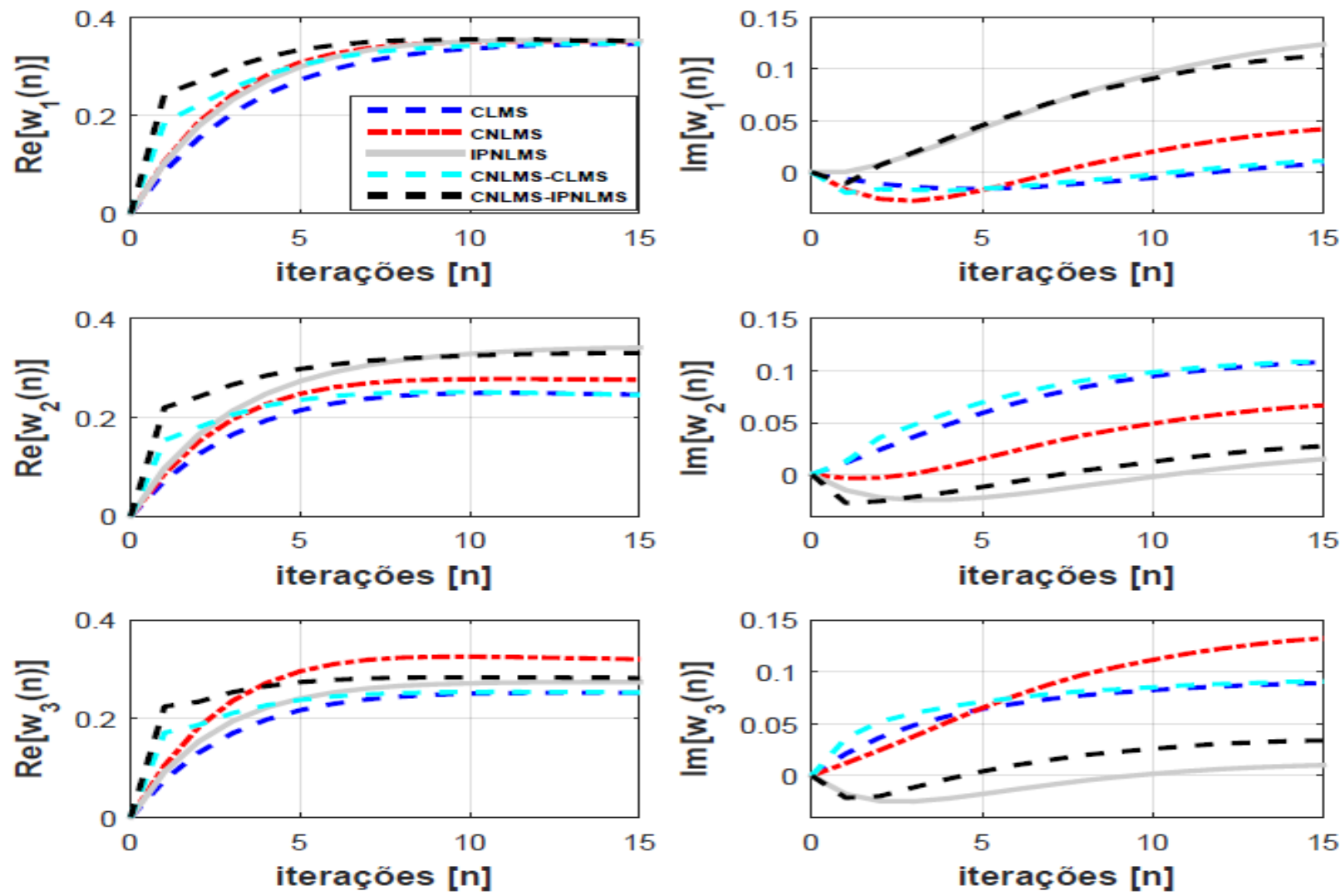

Fig. 3: Comportamento médio do vetor peso dos algoritmos de conformação de feixe convencionais e propostos para um arranjo adaptativo de 3 elementos na antena, com valores da parte real e complexa do $\Delta \mathrm{W}_{1-3}$ e SINR=20 dB.
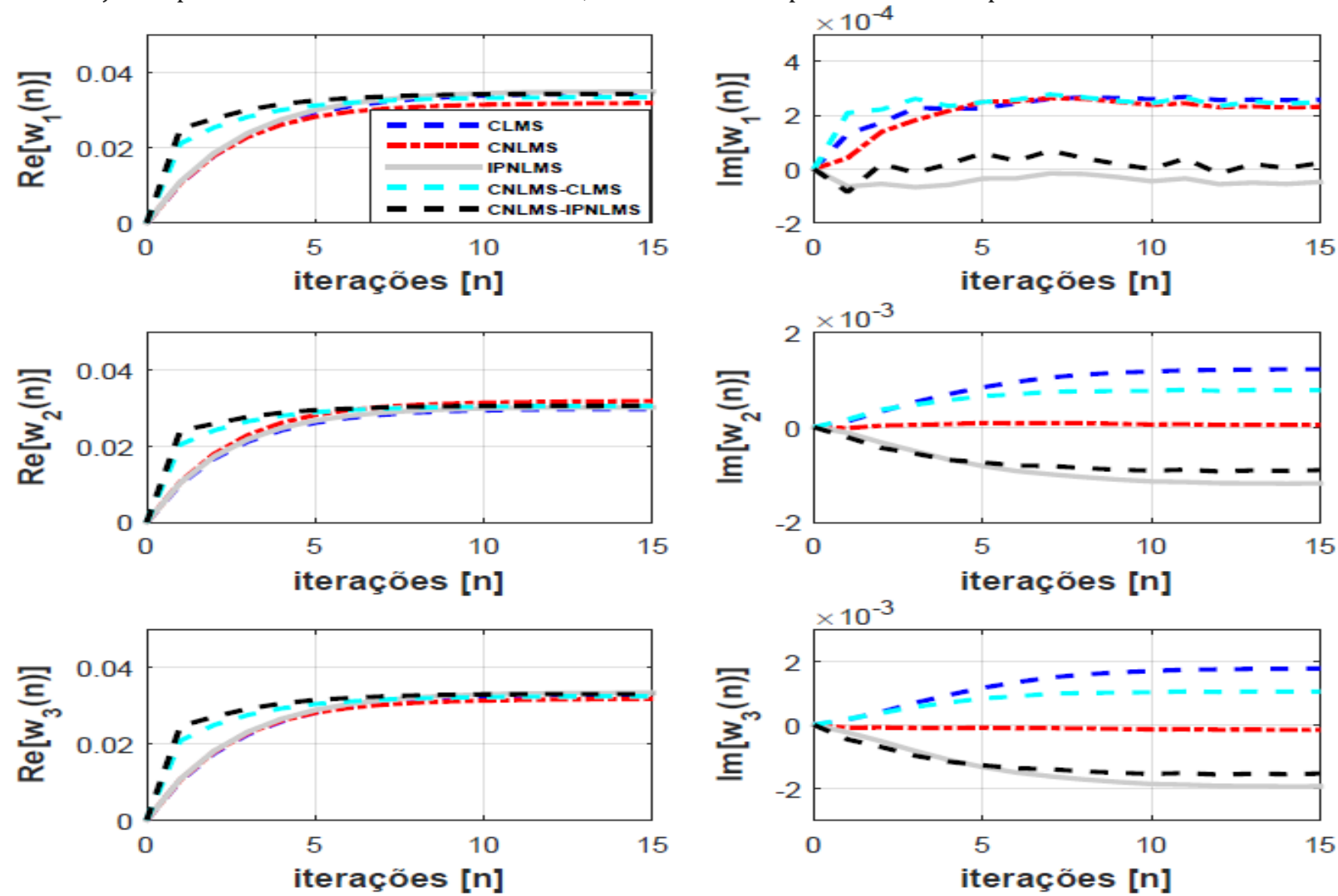

Fig. 4: Comportamento médio do vetor peso dos algoritmos de conformação de feixe convencionais e propostos para um arranjo adaptativo de 32 elementos na antena, com valores da parte real e complexa do $\Delta \mathrm{W}_{1-3}$ e SINR $=20 \mathrm{~dB}$. 

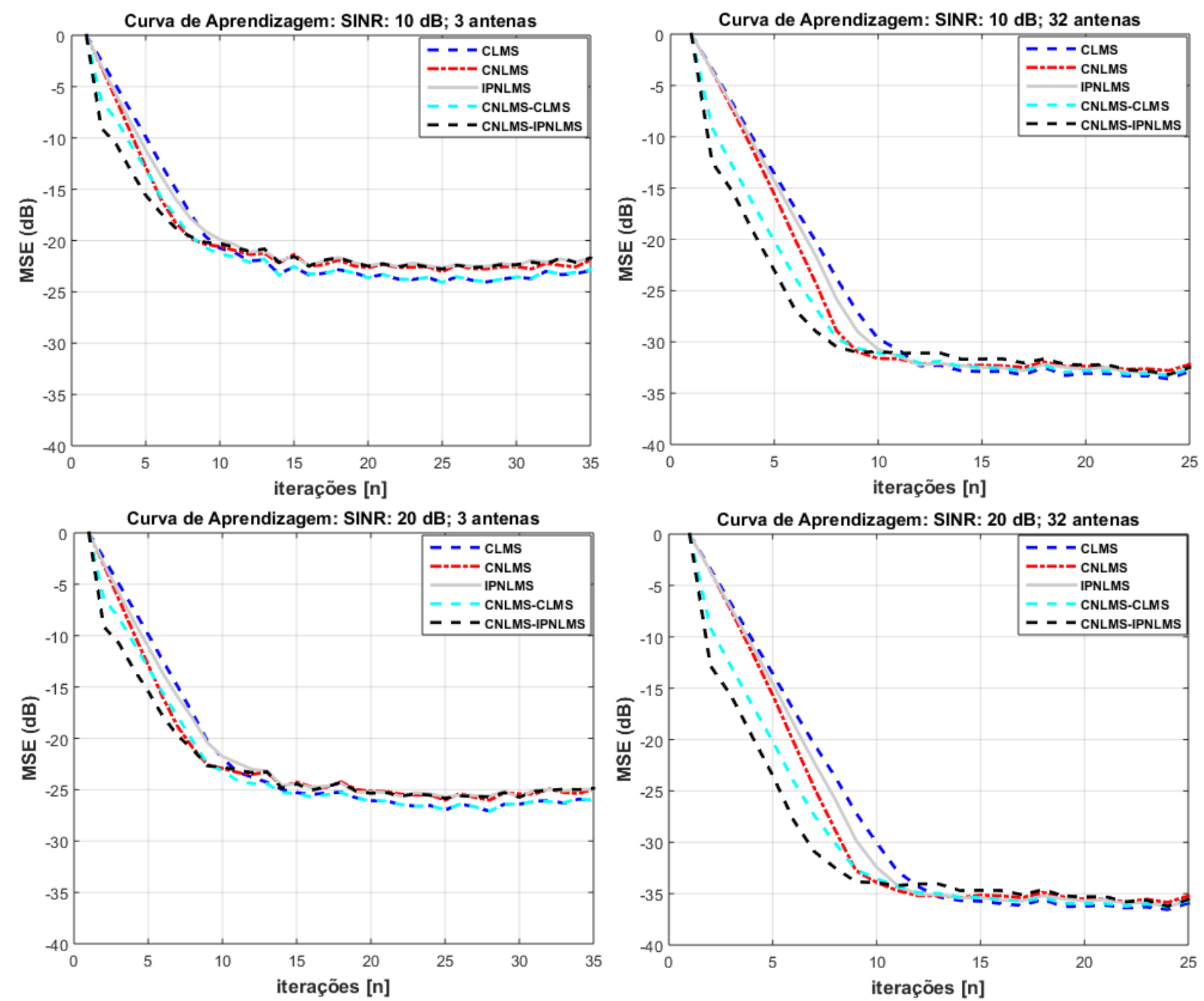

Fig. 5: Curvas de Aprendizagem dos algoritmos CLMS, CNLMS, IPNLMS, CNLMS-CLMS e CNLMS-IPNLMS.

\section{CONCLUSÕES}

Neste artigo, um filtro combinado convexamente foi proposto para aplicação em arranjo de antenas inteligentes. A metodologia proposta, baseia-se em uma combinação convexa de dois filtros adaptativos. Os pesos do arranjo adaptativo são obtidos combinando convexamente os pesos de cada algoritmo adaptativo da combinação usando um parâmetro de mistura, o qual é atualizado por um esquema de adaptação normalizada. Resultados de simulações, considerando dois cenários distintos (em termos de número de elementos do arranjo, SINR e tamanho de passo), mostram que a metodologia proposta apresenta maior velocidade de convergência em comparação a metodologias clássicas.

\section{AGRADECIMENTOS}

O presente trabalho foi realizado com apoio da Fundação de Amparo à Pesquisa e ao Desenvolvimento Científico do Maranhão (FAPEMA), da Coordenação de
Aperfeiçoamento de Pessoal de Nível Superior (CAPES) e Conselho Nacional de Desenvolvimento Científico e Tecnológico (CNPq).

\section{REFERÊNCIAS}

Alexiou, A., \& Haardt, M. (2004). Smart antenna technologies for future wireless systems: trends and challenges. IEEE communications Magazine, 42(9), 90-97.

Ali H. Sayed, Adaptive Filters. Hoboken, NJ: JohnWiley \& Sons, Apr. 2008.

Apolinário, J. A., Werner, S., Diniz, P. S., \& Laakso, T. I. (1998, September). Constrained normalized adaptive filters for CDMA mobile communications. In 9th European Signal Processing Conference (EUSIPCO 1998) (pp. 1-4). IEEE.

Arenas-Garcia, J., Azpicueta-Ruiz, L. A., Silva, M. T., Nascimento, V. H., \& Sayed, A. H. (2016). Combinations of adaptive filters: performance and convergence 
properties. IEEE Signal Processing Magazine, 33(1), 120-140.

Azpicueta-Ruiz, L. A., Figueiras-Vidal, A. R., \& ArenasGarcia, J. (2008, March). A normalized adaptation scheme for the convex combination of two adaptive filters. In 2008 IEEE International Conference on Acoustics, Speech and Signal Processing (pp. 33013304). IEEE.

Benesty, J., \& Gay, S. L. (2002, May). An improved PNLMS algorithm. In 2002 IEEE International conference on Acoustics, Speech, and Signal Processing (Vol. 2, pp. II1881). IEEE.

Boukalov, A. O., \& Haggman, S. G. (2000). System aspects of smart-antenna technology in cellular wireless communications-an overview. IEEE Transactions on Microwave Theory and Techniques, 48(6), 919-929.

Brandwood, D. H. (1983, February). A complex gradient operator and its application in adaptive array theory. In IEE Proceedings H-Microwaves, Optics and Antennas (Vol. 130, No. 1, pp. 11-16). IET.

de Andrade, J. F., de Campos, M. L., \& Apolinário, J. A. (2012, June). An L 1-norm linearly constrained LMS algorithm applied to adaptive beamforming. In 2012 IEEE 7th Sensor Array and Multichannel Signal Processing Workshop (SAM) (pp. 429-432). IEEE.

de Andrade, J. F., de Campos, M. L. R., \& Apolinário, J. A. (2013, May). An L1-constrained normalized Ims algorithm and its application to thinned adaptive antenna arrays. In 2013 IEEE International Conference on Acoustics, Speech and Signal Processing (pp. 3806-3810). IEEE.

de Andrade, J. F., de Campos, M. L., \& Apolinario, J. A. (2015). L1-Constrained Normalized LMS Algorithms for Adaptive Beamforming. IEEE Transactions on Signal Processing, 63(24), 6524-6539.
Frost, O. L. (1972). An algorithm for linearly constrained adaptive array processing. Proceedings of the IEEE, 60(8), 926-935.

Korayem, R., \& Bendoukha, S. (2018). A gradient descent implementation of the adaptive robust narrowband constrained LMS beamformer. Signal, Image and Video Processing, 12(3), 463-470.

Liu, L., Fukumoto, M., \& Zhang, S. (2008, November). A variable parameter improved proportionate normalized LMS algorithm. In APCCAS 2008-2008 IEEE Asia Pacific Conference on Circuits and Systems (pp. 201-204). IEEE.

Moghaddam, S. S., Moghaddam, M. S., \& Rad, R. K. (2010, July). A novel adaptive LMS-based algorithm considering relative velocity of source. In 2010 7th International Symposium on Communication Systems, Networks \& Digital Signal Processing (CSNDSP 2010) (pp. 10-14). IEEE.

Orozco-Tupacyupanqui, W., Nakano-Miyatake, M., \& PérezMeana, H. (2015, November). Adaptive hybrid beamforming based on a convex combination of complex stochastic gradient filters. In 2015 7th IEEE Latin-American Conference on Communications (LATINCOM) (pp. 1-6). IEEE.

Orozco-Tupacyupanqui, W., Carpio-Alemán, M., NakanoMiyatake, M., \& Pérez-Meana, H. (2016, November). Analysis of convex adaptive structures and algorithms for smart antennas. In 2016 IEEE International Autumn Meeting on Power, Electronics and Computing (ROPEC) (pp. 1-6). IEEE.

Paulo S. Diniz, Adaptive Filtering: Algorithms and Practical Implementation, Springer, 3rd edition, October 2010.

Srar, J. A., Chung, K. S., \& Mansour, A. (2010). Adaptive array beamforming using a combined LMS-LMS algorithm. IEEE Transactions on Antennas and Propagation, 58(11), 3545-3557. 